\title{
BACKWARD WHIRL OF UNBALANCED SHAFT AT ELASTICALLY CONSTRAINED JOURNAL BEARINGS AND FLOATING SEALS DUE TO MECHANICAL CONTACT \\ NELİDZSVAROTĀS VÄRPSTAS ATGRIEZENISKĀ PRECESIJA ELASTİGI \\ NOSTIPRINĀTAJOS SLIDDOŠAJOS GULTN̦OS UN PELDOŠAJOS SABLIIVEJUUMS MEHĀNISKĀ KONTAKTA REZULTĀTĀ
}

\author{
Ludmila Banakh, Andrey Nikiforov \\ Mechanical Engineering Research Institute of Russian Academy of Sciences \\ 4, M.Kharitonjevsky Lane, Moscow, 101990, Russia \\ Ph: +(7916)5226513, fax: +(7499)1353047, e-mail: n.andre@mail.ru
}

\begin{abstract}
The paper deals with the known phenomenon which takes place at rotor-stator contact. It is backward precession (whirl) of unbalanced shaft inside rigidly constrained sleeves (journal bearings) in the range below its critical speed. The purpose of this theoretical work is to research dynamic behavior of unbalanced shaft at the elastically constrained sleeves and above-resonant range.
\end{abstract}

Keywords: Contact rotation, dynamics and stability of high-speed rotor systems.

\section{Introduction}

The mechanical contact or rub between unbalanced shaft and journal bearing can produce two qualitatively various dynamic features $[1 ; 2]$.

Firstly, there is "lunar motion of shaft" at the condition of plentiful liquid lubrication. In this case the shaft rubs against the bearing by the same party, where the unbalance is located, while its points whirl in a direct direction (in the rotation direction) with the angular velocity which equals rotational frequency $\omega$.

Secondly, there is dynamic regime "backward whirl of shaft" in the ideal or sliding form at the conditions of poor or viscous lubrication. Ideal form arises at comparably low rotational frequency and then the shaft rolls on an internal surface of the journal bearing without sliding. In this case its center whirls on a circle of radius $\delta$ in direction opposite to rotation with constant angular velocity $\omega R / \delta$, where $R$ is shaft radius, $\delta$ is radial clearance, and anyone eccentric point of shaft describes the hypocycloids with number of loops which not depends on $\omega$ and equals $R / \delta$ i.e. the number of shaft precessions per its one revolution. For example, look at a result of one experiment where rotational frequency $\omega=300 \mathrm{rpm}$, shaft radius $R=6 \mathrm{~mm}$ and radial clearance $\delta=0.25 \mathrm{~mm}$ (Fig.1, photo 1). It shows the hypocycloid with number of loops $R / \delta=24$. The shaft can roll and with sliding then its whirl velocity becomes smaller than $\omega R / \delta$ and depends on $\omega t$. For example, the same experimental system at rotational frequency $\omega=600 \mathrm{rpm}$ has produced the hypocycloid with number of loops 7 (Fig.1, photo 2).

Field experience shows the regime "backward whirl of shaft" is most adverse for work. It conducts to fast heating and wear of journal bearing. Besides, the transition to such regime is accompanied by significant reduction of rpm (revolutions of shaft per minute), and restoration of former rotational frequency demands an expense of additional power. Reduction of revolutions number is connected to the arising resistance moment directed against the engine moment that approximately is determined by a ratio:

$$
I d \omega / d t=f N R,
$$

where $I-$ the total inertia moment of shaft and engine;

$N$ - normal pressure of shaft upon bearing. 
At regime "lunar motion of shaft" also there is a wear of journal bearing but not such intensive as at regime "backward whirl of shaft".

It is necessary to notice the given dynamic features correspond to unbalanced shaft whirling inside the rigidly constrained journal bearings in the range below its critical speed. Furthermore, at least according to author's data, there is no the direct knowledge for shaft whirl in case of elastically constrained journal bearings [3] or floating sealing rings [4]. It is unexpected as these elements for a long time are used correspondingly as supports and seals in the high-speed rotor systems (for example in the turbines).

(1)

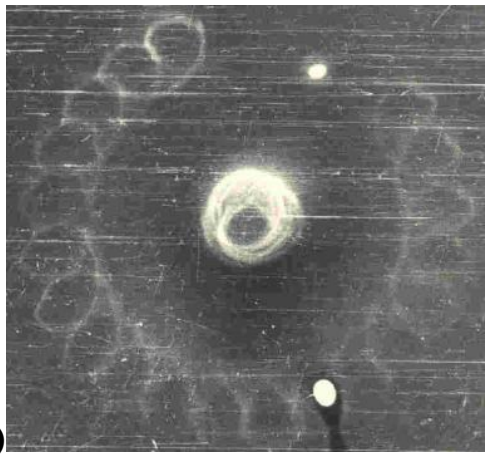

(2)

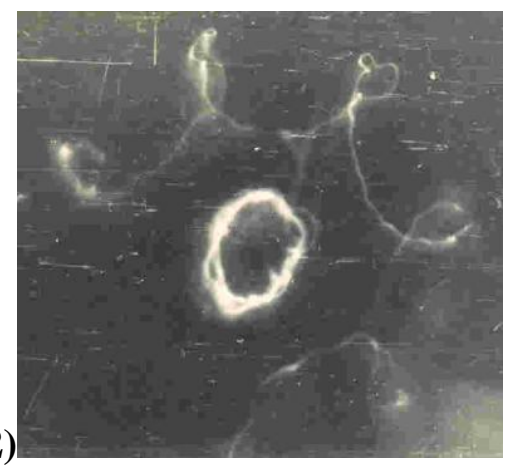

Fig. 1. Possible trajectories of eccentric point of shaft depending on $R / \delta$ и $\omega$

Elastically constrained journal bearings allow providing the small amplitudes of shaft oscillations and the low forces between shaft and bearings at the passage of critical speeds. Also they allow suppressing the shaft self-oscillations caused by properties of a lubricant layer.

Floating sealing rings are placed in the special frame niches stipulated in those places where it is necessary to seal the working fluids on constructive reasons. Very small annular clearances (as a rule less than $0.25 \mathrm{~mm}$ ) formed between shaft and rings provide an effect of the extremely limited leakage of liquid or gas. Leakage around of rings 1 is prevented by pressing of their face surfaces to the frame (Fig.2) due to a difference of pressure $\mathrm{P}_{1}>\mathrm{P}_{2}$ and the axial springs 3 entered in case of small working pressure. The pins 4 not limiting radial mobility of rings (Fig.2, a) are usually used in order to the friction surface 2 worked without relative rotation.

a)

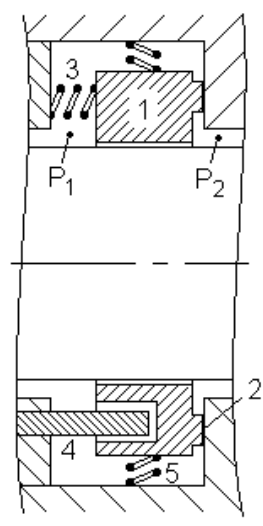

b)

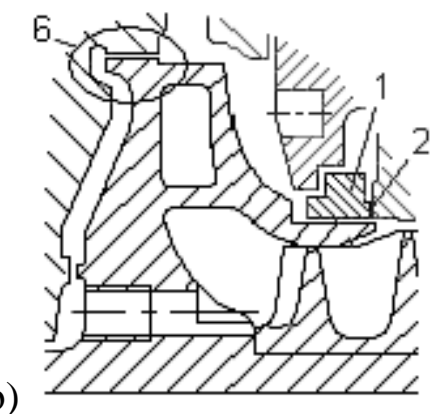

Fig. 2. Floating sealing ring 1 of shaft (a) and impeller (b)

Advantage of floating sealing ring in comparison with static seal 6 (Fig.2, b) or rigidly constrained sleeve consists in the next. Ring is self-aligning in radial direction during work concerning the shaft surface tracing all its motions. It allows creating a smaller annular clearance for reduction of leakage and it allows sealing a vibrating shaft. 
Fundamental work principle of floating ring is a performance of the self-centering. It is a condition when the maximal hydro(gas)dynamic force in annular clearance exceeds both the friction force on contact surface and the elastic force of radial springs 5 in some designs. However if this condition is not performed the ring can easily change the position under action of shaft impulses. Such rings rank between floating rings and static seals.

\section{Simulation of researched system}

Taking into account the denoted problem, the technical and dynamic features for application of elastically constrained journal bearings and floating sealing rings, the following system was considered (Fig.3). It is a vertical rotor by the mass $m$ with unbalanced disk located in the middle of rigid shaft. It is supported by two identical gapless bearings positioned symmetrically, each of which has rigidity $k / 2$ and equivalent coefficient of damping $d / 2$. Besides the shaft is supported by two identical sleeves constrained elastically, each of which has radial clearance $\delta$, mass $m_{0} / 2$, the suspension bracket by rigidity $k_{0} / 2$ and equivalent coefficient of damping $d_{0} / 2$.

Let's notice the value $d_{0}$ allows taking into account not only the viscous friction of sleeves induced by an environment but also - the dry friction of sleeves on the frame (in case of sealing rings). It is possible to put $d_{0}=4 F_{\mathrm{fr}} /(\pi \Omega A)$, where $F_{\mathrm{fr}}$ is friction force between rubbing surfaces, $\Omega$ is frequency of sleeve precession and $A$ is amplitude of sleeve precession.
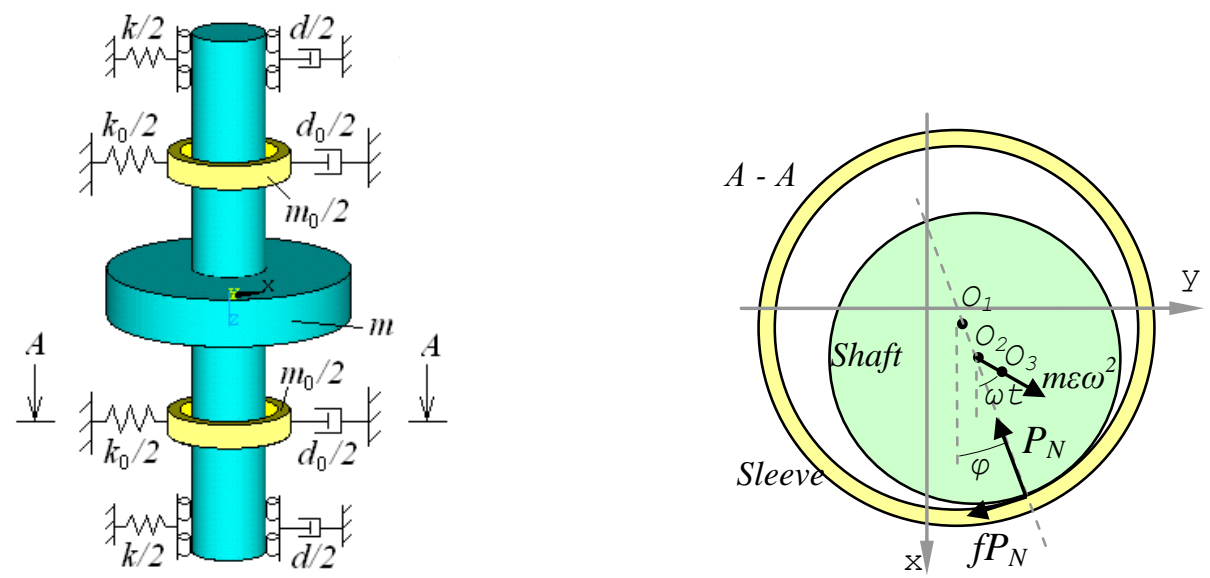

Fig. 3. Calculated model of researched system

The motion equations of accepted system were written down in the Cartesian coordinates and at a few assumptions. Fluid reactions in the sleeve clearance is ignoring because they is small in comparison with external forces $\left(k_{0} x_{1}, k_{0} y_{1}, d_{0} x_{1}, d_{0} y_{1}\right)$. Shaft speed $\omega$ is a constant due to the powerful engine. Disk motion is flat. Motion of the sleeves centers is identical due to the symmetry. Let the next be in this system: $x_{1}, y_{1}$ are coordinates for the centre of sleeve $O_{1}$ (for top sleeve and for lower sleeve), $x_{2}, y_{2}$ are coordinates for the centre of shaft $O_{2}, x_{3}, y_{3}$ are coordinates for the mass centre of shaft $\mathrm{O}_{3}, \varepsilon$ is rotor eccentricity $\left(\varepsilon=\mathrm{O}_{2} \mathrm{O}_{3}\right), \varphi$ is angle for the line of the centers (is angle between a relative displacement $e=O_{1} O_{2}$ and an axis $x$ ).

Let's express contact reaction of sleeves and shaft as components $P_{N}$ and $f P_{N}$, and also let's take into account that $x_{3}=x_{2}+\varepsilon \cos \omega t$ and $y_{3}=y_{2}+\varepsilon \sin \omega t$. In view of the accepted assumptions the differential motion equations of system are defined as follows:

$$
\left\{\begin{array}{l}
m_{0} \ddot{x}_{1}+d_{0} \dot{x}_{1}+k_{0} x_{1}-\left(P_{N} \cos \varphi-f P_{N} \sin \varphi\right)=0, \\
m_{0} \ddot{y}_{1}+d_{0} \dot{y}_{1}+k_{0} y_{1}-\left(P_{N} \sin \varphi+f P_{N} \cos \varphi\right)=0, \\
m \ddot{x}_{2}+d \dot{x}_{2}+k x_{2}+\left(P_{N} \cos \varphi-f P_{N} \sin \varphi\right)=m \varepsilon \omega^{2} \cos \omega t, \\
m \ddot{y}_{2}+d \dot{y}_{2}+k y_{2}+\left(P_{N} \sin \varphi+f P_{N} \cos \varphi\right)=m \varepsilon \omega^{2} \sin \omega t .
\end{array}\right.
$$


The first two equations of system (1) describe oscillations of the sleeve under action of elastic forces $k_{0} x_{1}, k_{0} y_{1}$, damping forces $d_{0} x_{1}, d_{0} y_{1}$ and forces of contact reaction $P_{N}$ and $f P_{N}$, directed opposite represented forces in Fig.3. The second two equations are close to considered in [5; 6] and they describe motion of shaft under action of centrifugal forces $m \varepsilon \omega^{2} \cos \omega t$, $m \varepsilon \omega^{2} \sin \omega t$, forces of contact reaction $P_{N}, f P_{N}$, elastic forces $k x_{2}, k y_{2}$ and forces of external damping $d \dot{x}_{2}, d \dot{y}_{2}$.

If the purpose of the dynamic analysis is not reception of quantitative results and only it is required the revealing of qualitative laws, the problem of contact motion both shaft and bearings/seals can be studied loosely (in the different hypothesises) concerning reaction $P_{N}$. Only it is necessary to notice, any such reaction should go to zero at the contact absence $(e<\delta)$ and it should grow with increase $e$, by other words for any function $P_{N}$ a condition should be satisfied: $P_{N}=0$ for $e<\delta, d P_{N} / d e>0$ for $e>\delta$. Besides, it is assumed the second derivative $d^{2} P_{N}$ $/ d e^{2}$ is continuous for $e>\delta$.

These requirements may be satisfied by the normal nonlinear Hertz's reaction $P_{N}=b \mathrm{e}^{3 / 2}$, where $b$ is coefficient dependent on curvature of bodies surfaces at the contact domain and $b$ depends on elastic properties of a material, e is an approaching between the centers of contacted bodies which has negligible deformations in comparison with their local deformations [7]. Accepting on analogy with [6] that the relative displacement of shaft and sleeve $e$ is value of their local deformations e, the definition for $P_{N}$ can be expressed by following equation:

$$
P_{N}=\left\{\begin{array}{l}
0, \quad(e<\delta), \\
k_{\mathrm{c}} e^{3 / 2}, \quad(e>\delta) .
\end{array}\right.
$$

In order to the equations (1) and (2) formed the closed loop system it is necessary to express relative displacement $e$ and angle $\varphi$ through the coordinates of sleeve $x_{1}, y_{1}$ and the coordinates of shaft $x_{2}, y_{2}$. Fig. 3 allows defining $x_{2}-x_{1}=e \cos \varphi$ and $y_{2}-y_{1}=e \sin \varphi$ and consequently:

$$
\begin{aligned}
& e=\sqrt{\left(x_{2}-x_{1}\right)^{2}+\left(y_{2}-y_{1}\right)^{2}}, \\
& \cos \varphi=\frac{x_{2}-x_{1}}{e}, \quad \sin \varphi=\frac{y_{2}-y_{1}}{e} .
\end{aligned}
$$

Integration of the equations (2-4) in the closed loop form is connected to the big mathematical difficulties and it is possible only with the help of a computer. Numerical calculations via Runge-Kutta method at the system parameters $m=1.5 \mathrm{~kg}, \varepsilon=10 \mu \mathrm{m}, k=1500 \mathrm{~N} / \mathrm{m}, d=d_{0}=10 \mathrm{Ns} / \mathrm{m}$, $m_{0}=0.2 m$, contact stiffness $k_{\mathrm{c}}=15000 \mathrm{~N} / \mathrm{m}$ and at the changing of friction coefficient $f$, rotational frequency $\omega$, radial clearance $\delta$ and rigidity $k_{0}$ were done.

\section{Results and conclusions}

1. At the extreme case $f=1$ and it was expected a priori, the regime "backward whirl of shaft" on an internal surface of the sleeve in the ideal form is obtained. Under the critical speed $\left(\omega<\omega_{0}=\sqrt{k / m}\right)$ the mass centre or any another eccentric point of shaft whirls in the rotation direction, making characteristic hypocycloids with finite number of loops $n=n\left(\delta, \omega . k_{0}\right)$ due to superposition of two circular motions at angular velocities $\omega$ and $-\Omega=n \omega$, while the central point of shaft whirls opposite to rotation on the circular orbit at frequency $\Omega$.

However over the critical speed $\left(\omega>\omega_{0}\right)$ there is a revolution in metaphorical and in literal sense due to the self-centering phenomenon of shaft. The mass centre of shaft receives the backward whirl at frequency $\Omega=\omega / n$, while the geometrical centre of shaft has direct hypocycloid whirl formed by motions at angular velocities $\omega$ and $-\omega / n$. Particularly, it is visible on displacements of points $\mathrm{O}_{2}$ and $\mathrm{O}_{3}$ from their initial states, on the display of oscillations along one axis and on the frequency spectrum (Fig.4). The displacements of point 
$O_{1}$ and its trajectories 1 allow to draw a conclusion the contact motion generates backward whirl of the sleeve centre at angular velocity $\Omega=n \omega$, when rotational frequency is below critical speed of shaft, or $\Omega=\omega / n$, when $\omega>\omega_{0}$ (Fig.4).
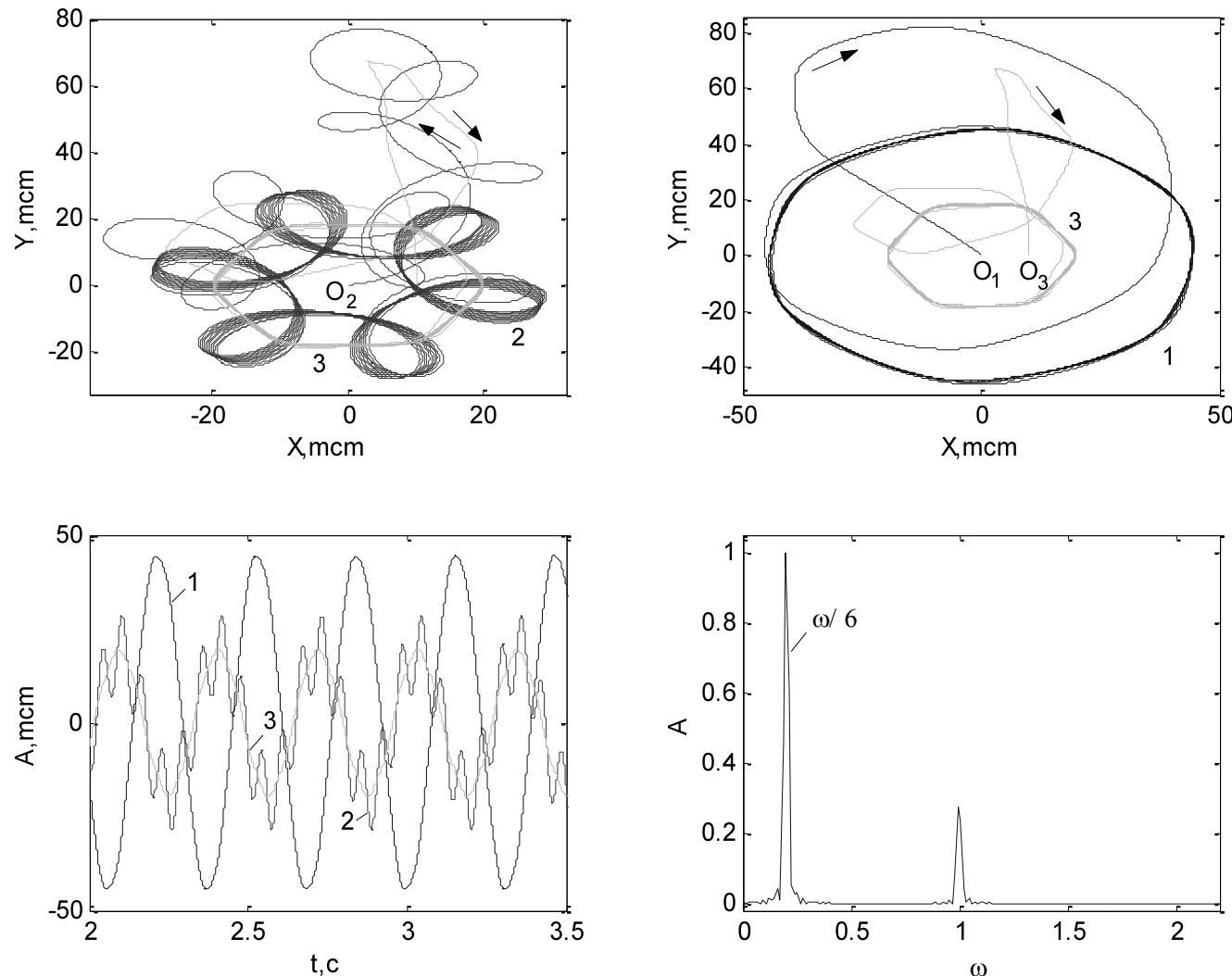

Fig. 4. Displacements of centres of sleeve 1 and shaft 2 and mass centre of shaft 3 , frequency spectrum for motion of centre 2 at $f=1, \omega=1000 \mathrm{rpm}, \delta=55 \mu \mathrm{m}, k_{0}=0$

2. At the other extreme case $f=0$ there is a usual at the unbalance, direct, synchronous whirl of shaft or any its points all over the range $\omega$. At the same time the sleeve performs a reciprocal motion at frequency $\omega$ due to periodic contacts between its diametric-opposite points and shaft.

3. For the middle values $f$ there is a slip to a greater or lesser extent. A battle of tendencies in dynamics of shaft and sleeve appears.
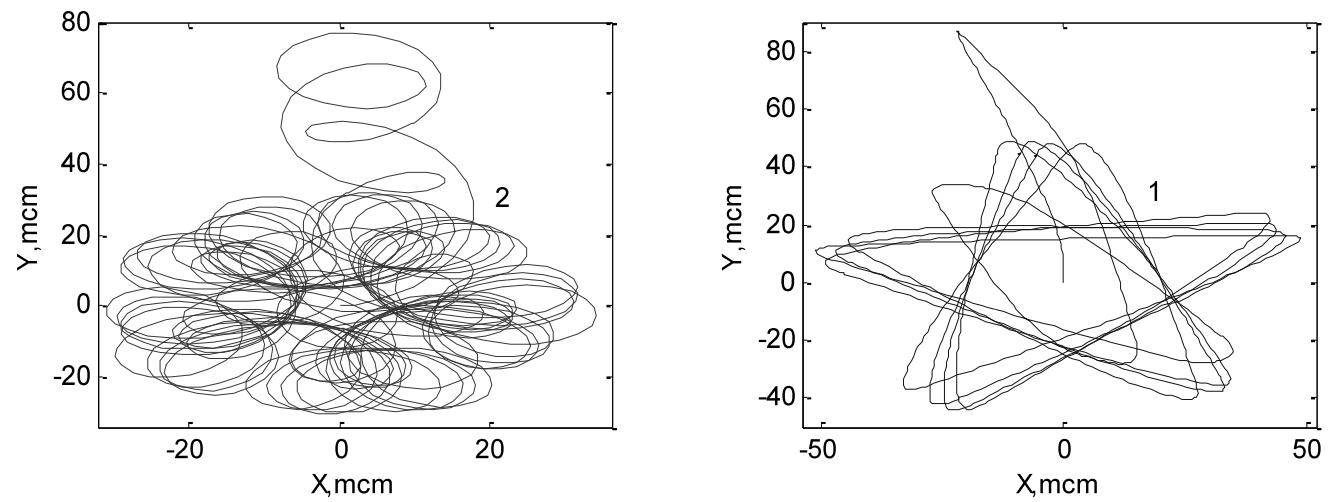

Fig. 5. Motions of sleeve 1 and shaft 2 centres at $f=0.35, \omega=1000 \mathrm{rpm}, \delta=55 \mu \mathrm{m}, k_{0}=0$ 
If $f<0.35$ the tendency No2 gains the upper hand, as consequence the shaft centre reproduces direct circular motion at angular speed $\omega$, and the sleeve centre describes $n$-pointed stars at the same frequency (Fig.5) and it is close to results [8]. If $f>0.35$ the tendency No1 prevails, as a result the backward whirl of centers of shaft and sleeve at the angular velocities unequaled $\omega$ takes place.

4. If radial clearance $\delta$ is less than the defined critical value $\delta_{\mathrm{cr}}=\delta_{\mathrm{cr}}(\omega)$ the hypocycloid whirl of shaft inside of sleeves does not arise, their lunar (direct synchronous) whirl is occurred (Fig.6).
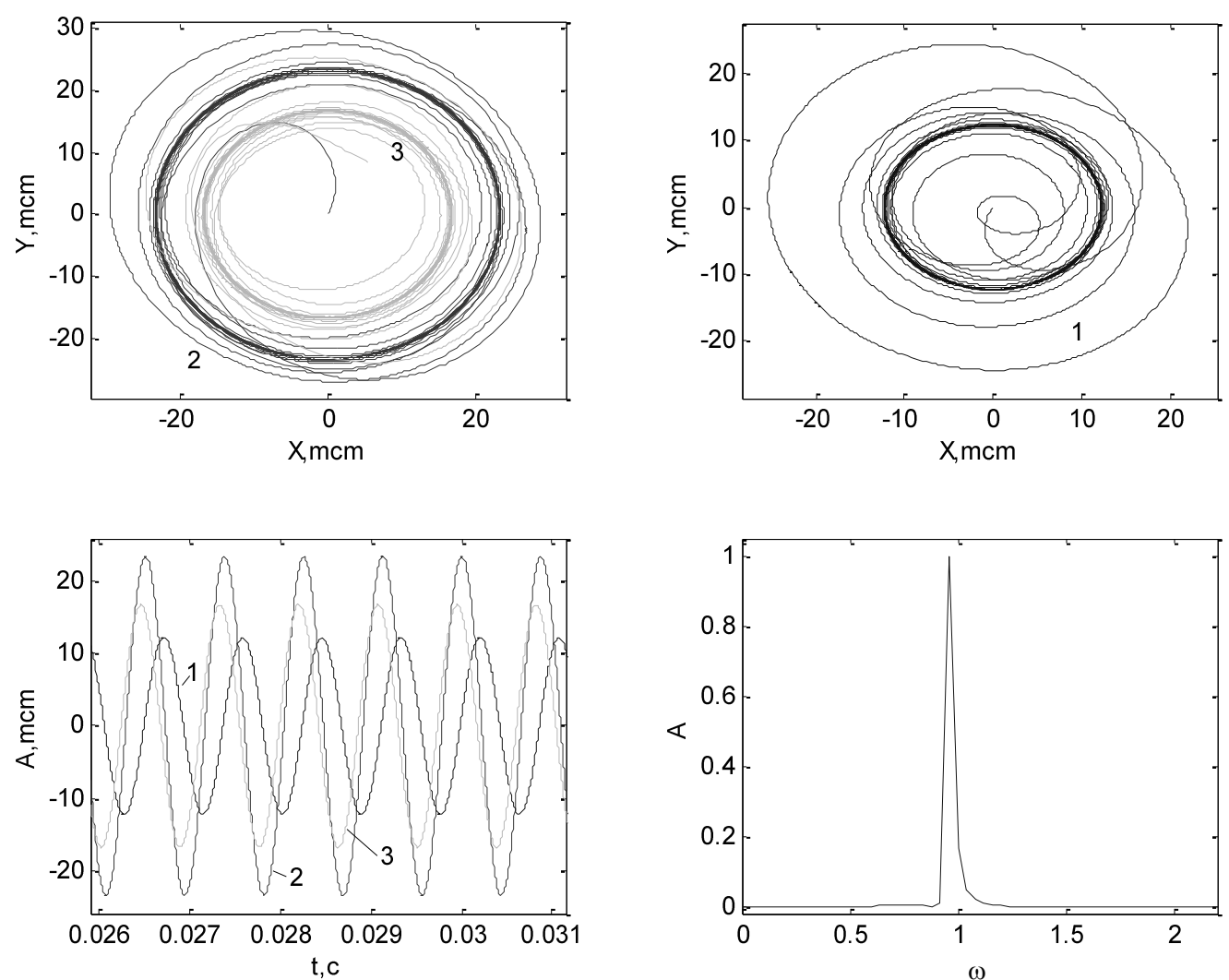

Fig. 6. Motions of researched system at $f=0.35, \omega=1000 \mathrm{rpm}, \delta=30 \mu \mathrm{m}, k_{0}=0$

5. As against a frequencies range of below critical speed, in the above resonance domain the number of loops $n$ grows at the increase $\omega$ (Fig.7, a). Besides, the number $n$ grows at the increase $k_{0}$ (Fig.7, b).

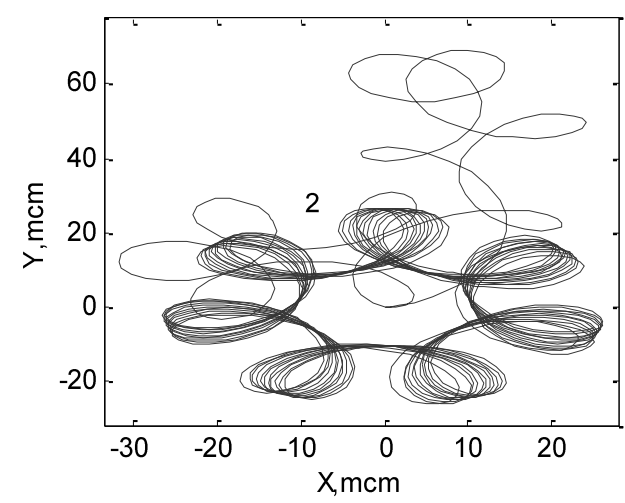

a)

Fig. 7. Motions of shaft centre a) at $f=1, \omega=1200 \mathrm{rpm}, \delta=55 \mu \mathrm{m}, k_{0}=0$

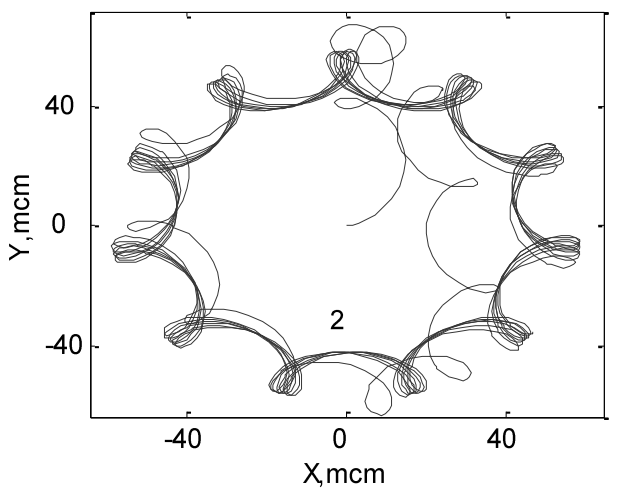

b) at $f=1, \omega=1200 \mathrm{rpm}, \delta=55 \mu \mathrm{m}, k_{0}=10^{3} \mathrm{~N} / \mathrm{m}$ 


\section{Summary}

Dynamics of unbalanced shaft in case of elastically constrained journal bearings and seals at the contact was researched. Dynamic analysis of that system for pre-resonant and aboveresonant speeds of rotation was fulfilled. Now a few interesting dynamic features for similar systems are known. Further scientific inquiry will be devoted to the contact dynamics between unbalanced shaft and free-rotating sleeves.

\section{Bibliography}

1. Banakh L. Some phenomena arising at the rotation of shaft on journal bearings // M.: Mashinovedenie, 1965. No1, p. 5-12.

2. Bartha A.R.: Dry Friction Induced Backward Whirl: Theory and Experiments, Fifth International IFToMM Conference on Rotor Dynamics, Darmstadt, Germany, 1998. p.755-767.

3. Keljzon A.S., Tsimansky Yu.P., Yakovlev V.I. Dynamics of rotors on elastically constrained bearings. M.: Nauka, 1982. 280 p.

4. Martsinkovsky V.A. Clearance seals of rotor machines. M.: Mashinostroenie, 1980. 200 p.

5. Edwards S., Lees A.W. and Friswell M.I. The Influence of Torsion on Rotor/Stator Contact in Rotating Machinery, Journal of Sound and Vibration, 1999. p. 767-778.

6. Braut S., Zigulic R., Skoblar A., Stimac G., Butkovic M., Jokic M. Dynamic Analysis of the Rotor-Stator Contact due to Blade Loss. Proc. 12 ${ }^{\text {th }}$ IFToMM World Congress, Besançon (France), 2007.

7. Biderman V.L. Calculation on impulsive loads. Handbook of engineering worker. M.: MASHGIZ, 1962. Vol. No3, p. 430-442.

8. Banakh L., Nikiforov A., Panovko G. Vibroimpact Motion of Rotor taking into account Friction at the Contact. Proc. $6^{\text {th }}$ EUROMECH Nonlinear Dynamics Conference (ENOC 2008), Saint Petersburg (Russia), 2008., online at http://lib.physcon.ru/?item=1605 